\title{
Patrimoine littéraire et éducation à la fraternité à l'école
}

Aldo Gennaï

\section{(2) OpenEdition \\ 1 Journals}

\section{Electronic version}

URL: http://journals.openedition.org/trema/5736

DOI: 10.4000/trema.5736

ISSN: 2107-0997

\section{Publisher}

Faculté d'Éducation de l'université de Montpellier

\section{Electronic reference}

Aldo Gennaï, « Patrimoine littéraire et éducation à la fraternité à l'école », Tréma [Online], 53 | 2020, Online since 01 March 2020, connection on 04 March 2020. URL : http://journals.openedition.org/ trema/5736 ; DOI : https://doi.org/10.4000/trema.5736

This text was automatically generated on 4 March 2020

Trema 


\title{
Patrimoine littéraire et éducation à la fraternité à l'école
}

\author{
Aldo Gennaï
}

1 La notion de fraternité (ou d'adelphité, pour utiliser le terme non genré proposé par Christine Bard, 2010, p. 379) implique étymologiquement la filiation et une ascendance commune. La fraternité, écrit Guillaume de Tanoüarn, « se reçoit, elle est l'héritage des siècles » $(2018$, p. 20$)$; elle procède « d'un sentiment de filiation qui est commun aux frères et aux sœurs» (Ibid., p. 21) et suppose «la reconnaissance d'une paternité commune » (Ibid., p. 22).

2 En ce sens, la fraternité peut être reliée à la notion de patrimoine, le terme désignant d'abord «l'ensemble des biens, des droits hérités du père » avant de prendre sa valeur générale : «ce qui est transmis à une personne, à une collectivité par les ancêtres, les générations précédentes ", en particulier, dans un emploi métaphorique, "les biens matériels et intellectuels hérités par une communauté » (Rey, 1992, p. 1452). Envisagée largement et idéalement comme le «lien entre des personnes se considérant comme appartenant à la famille humaine » (Ibid., p. 841), la fraternité pourrait donc se fonder sur le sentiment d'une paternité (c'est-à-dire d'une origine et d'une condition) commune(s), et d'un héritage, d'un patrimoine partagé.

3 Les œuvres littéraires faisant partie de ce legs, on peut se demander dans quelle mesure l'enseignement des textes patrimoniaux à l'école est susceptible de contribuer à une éducation à la fraternité, entendue comme le sentiment d'une appartenance fondamentalement commune à la vaste famille humaine, au-delà ou en-deçà des particularismes et des différences interindividuelles. En quoi et comment la lecture et l'étude de ces œuvres peuvent-elles inspirer et faire éprouver ce sentiment de fraternité ? Quels dispositifs pédagogiques peut-on mettre en œuvre dans les classes à cette fin?

4 J'aborderai ces questions selon une perspective essentiellement théorique et spéculative (qu'il faudrait donc mettre à l'épreuve des résultats de recherches empiriques) en m'appuyant sur des travaux relativement récents, également théoriques et conceptuels, menés dans le champ de la didactique de la littérature, et qui 
interrogent l'objet patrimonial, sa définition, la constitution du canon des œuvres dites classiques ou patrimoniales, leur enseignement, et les phénomènes qui assurent leur conservation et leur transmission (Ahr \& Denizot, 2013; Bishop \& Belhadjin, 2015; de Peretti \& Ferrier, 2012). Pour illustrer et alimenter ma réflexion, je ferai occasionnellement référence à une séquence consacrée à l'étude du conte La Belle et la Bête (1756), observée, dans le cadre d'un GRIF (Groupe Recherche Innovation Formation) de l'Université de Montpellier sur l'enseignement de la littérature patrimoniale, dans une classe de CM2 d'une école nîmoise ${ }^{1}$, entre septembre 2015 et mai 2016. Les données recueillies à l'occasion de cette action sont constituées de réponses à des questionnaires écrits adressés aux élèves et à leurs parents et d'enregistrements audiovisuels de trois séances de classe. Le conte de Jeanne-Marie Leprince de Beaumont, inscrit dans les listes de référence pour le cycle 3, peut permettre d'engager une réflexion en classe sur les apparences, souvent trompeuses comme on le sait, sur les rivalités au sein de la fratrie, l'altérité, le respect des différences, etc. Cependant, dans la perspective qui est la mienne, c'est davantage le fort indice de patrimonialité de l'œuvre, évalué à l'aune des discours variés qu'elle continue de générer, qui me parait intéressant, mon postulat étant le suivant: il ne s'agit pas tant, pour éduquer à la fraternité, d'étudier une œuvre patrimoniale au cœur de laquelle ce thème serait inscrit, que d'éprouver la patrimonialité dans ses œuvres et pour ainsi dire à l'œuvre.

\section{Valeurs dans les textes patrimoniaux ou valeur $d u$ phénomène patrimonial ?}

5 Le patrimoine littéraire est fondamentalement une affaire de valeur(s). D'une part, il constitue par définition un bien précieux, un héritage commun à conserver et à valoriser, pour sa valeur historique et ses qualités esthétiques éminentes. Comme l'écrit Nathalie Heinich,

le «patrimoine » est en lui-même une catégorie valorisée, de sorte que le simple fait de catégoriser un artefact comme patrimonial [...] signifie qu'on lui accorde une valeur, quelle qu'elle soit, et quelle que soit la façon dont, concrètement, cette valorisation se manifeste (Heinich, 2009, p. 151).

D'autre part, le patrimoine littéraire est volontiers considéré comme un grand « pourvoyeur de valeurs et de références » (Bishop \& Belhadjin, 2015, p. 21).

\section{Des textes patrimoniaux exemplaires pour éduquer à la fraternité ?}

7 Il importe alors en tant qu'il est constitué de textes où sont inscrites des valeurs qu'il véhicule et illustre. Cette foi dans les vertus éducatives des œuvres patrimoniales s'exprime déjà dans les programmes du 27 juillet 1882 de Jules Ferry :

il s'agit de donner une véritable culture aux élèves des écoles primaires au-delà du simple lire-écrire-compter, grâce à la connaissance des textes du patrimoine littéraire français. L'objectif est de développer un fonds commun d'idées, de représentations et de valeurs laïques ${ }^{2}$ que la littérature contribue à élaborer (Bishop, 2015, p. 370).

8 Aujourd'hui encore, la notion de patrimoine revêt des enjeux éducatifs et idéologiques forts, souvent en lien, dans les débats politiques et médiatiques contemporains, avec la question de l'identité et de la cohésion nationales. L'institution scolaire même semble 
continuer à considérer que l'enseignement de la littérature patrimoniale peut être mis au service de la défense, de l'illustration comme de la diffusion de valeurs républicaines et des idéaux de liberté, d'égalité et de fraternité. Par exemple, les propositions bibliographiques publiées en janvier 2015 par le ministère de l'Éducation nationale, après les attaques terroristes, pour aborder la question de la liberté de conscience et d'expression, témoignent d'un fort tropisme patrimonial : la moitié des vingt textes retenus pour le cycle 3 appartiennent au patrimoine littéraire français (il s'agit de dix fables de La Fontaine). Ce document témoigne que la tentation est toujours forte de s'en remettre au patrimoine littéraire national dans la tourmente et de considérer ces œuvres comme un mode d'expression et de défense privilégié des valeurs humanistes menacées par l'obscurantisme. Une société est d'autant plus attachée à son patrimoine qu'elle estime, à tort ou à raison, que ce patrimoine, la culture et les valeurs qu'il incarne sont menacés; en situation de crise, lorsque les valeurs (morales, humanistes, républicaines...) semblent en danger, le patrimoine littéraire est revalorisé.

Quoi qu'il en soit, « l'œuvre patrimoniale est celle dont tous les élèves doivent hériter, le bien commun, fondant une culture commune» (Houdart-Merot, 2012, p. 35). Le patrimoine littéraire est perçu comme propre à s'opposer aux particularismes et à « rassembler les citoyens autour d'une même culture dont l'École serait porteuse " (Bishop \& Belhadjin, 2015, p.25) - au risque d'une conception normative des enseignements moraux et civiques à tirer de la littérature, envisagée alors comme un réservoir de comportements exemplaires et de leçons de morale univoques (Gennaï, 2018b).

\section{Fonder l'éducation à la fraternité sur le processus patrimonial}

10 Sans nier l'intérêt que peuvent présenter les œuvres du patrimoine au service d'une réflexion à orientation morale et civique, il me semble que, non moins que son matériau thématique et axiologique, c'est la nature patrimoniale même de l'œuvre qui peut contribuer à une éducation à la fraternité. Le processus me parait importer davantage que le produit.

11 La fabrique du patrimoine littéraire repose en effet sur un «double mouvement de réception-transmission» (Denizot, 2015, p. 110) ; le patrimoine est un bien hérité des générations passées, qui ont elles-mêmes reçu, conservé, accru et reconfiguré ce capital culturel, et nous l'ont transmis, en nous chargeant d'en assurer à notre tour la conservation et la transmission. Au service d'une éducation à la fraternité, c'est donc le phénomène et la notion mêmes de patrimonialité qui peuvent être interrogés, afin d'en faire éprouver aux élèves la dynamique essentielle de conservation et de transmission d'un héritage commun.

12 Il s'agit ainsi de faire percevoir le partage au cœur du fait patrimonial, partage devant être entendu dans la seconde des deux acceptions du terme signalées par Jacques Derrida :

ce mot partage [...] en français nomme aussi bien la différence, la ligne de démarcation ou le partage des eaux, la scission, la césure que, d'autre part, la participation, ce qu'on partage parce qu'on y communique ou l'a en commun, au titre de l'appartenance » (Derrida, 1986, p. 59). 
Il s'agit de faire percevoir la faculté de circulation des textes du patrimoine, notamment dans l'espace intergénérationnel, comme un symbole, au sens étymologique du terme ${ }^{3}$ :

Le latin [symbolus] reprend le grec sumbolon, désignant un signe de reconnaissance,

à l'origine un objet coupé en deux dont deux hôtes conservaient chacun une moitié qu'ils transmettaient à leurs enfants (Rey, 1992, p. 2062).

La fonction symbolique du patrimoine est alors de fonder et d'attester l'appartenance à une communauté, de permettre de se reconnaitre et reconnaitre autrui comme membres de cette communauté.

\section{Le patrimoine : du « passé présent »}

D'un point de vue juridique, les œuvres du patrimoine sont des œuvres libres de droits (Louichon, 2015a, p. 94). On peut retenir avec Brigitte Louichon une autre définition et considérer l'œuvre patrimoniale comme du "passé présent", à la fois "production passée et réception présente" (Louichon, 2015b), cette réception continuant de s'opérer au présent grâce au " nuage de discours » (Calvino, 1991, p. 9) que suscitent les textes du patrimoine littéraire. L'œuvre patrimoniale «se caractérise par sa présence effective, par son actualité réelle ", assurées par sa faculté à générer, dans des sphères médiatiques diverses et sous des formes variées, des " objets sémiotiques secondaires " ou OSS qui les escortent et signent leur patrimonialité (Louichon, 2015a, p. 100). Outre les hypertextes (parodies, pastiches, «transfictions ${ }^{4}$ ), les métatextes («les commentaires, les discours critiques, les discours historiographiques, les discours scolaires, les discours autobiographiques qui disent quelque chose du texte»; ibid., p. 102) et les allusions ("une modalité de coprésence entre deux textes»; idem) qu'engendrent les textes $\mathrm{du}$ patrimoine, les objets sémiotiques secondaires comprennent également les adaptations: éditions, traductions inter- et intralinguistiques, et toutes les formes de réécriture relevant de la multimodalité (combinaison de différents modes sémiotiques) et de la transmédialité (migration d'un art ou d'un médium vers un autre, de l'écrit à l'écran par exemple).

16 Si les nombreuses adaptations qui escortent l'œuvre patrimoniale et composent une « littérature au second degré » (Genette, 1982) sont nécessairement secondes au sens chronologique du terme (elles sont également secondaires dans la hiérarchie des biens culturels, surtout lorsqu'il s'agit de produits de consommation de masse tels que les dessins animés des studios Disney, les mangas et les jeux vidéo), ces OSS sont souvent premiers dans le processus par lequel s'opèrent la réception et la transmission du patrimoine littéraire. Ils fondent une première connaissance de l'œuvre patrimoniale par les élèves et jouent un rôle non négligeable dans la constitution d'une première culture littéraire et artistique commune (Gennaï, 2018a). Il me semble qu'on peut accueillir en classe les OSS connus des élèves et les inscrire au cœur d'un dispositif pédagogique permettant de leur faire éprouver la fonction symbolique du patrimoine et la manière dont ces textes participent à la construction du commun indispensable pour faire société. 


\section{Quelques propositions}

17 Sur quel(s) usage(s), quelle(s) pratique(s) des œuvres patrimoniales accompagnées de leurs OSS fonder une éducation à la fraternité à l'école ? Insistons-y, il ne suffit pas de convoquer ces textes dans les classes, de les lire et d'en débattre pour activer leur vertu éducative, dans la mesure où

Les valeurs ne sont pas un dépôt inscrit dans les œuvres comme quelque chose de figé et qu'il faudrait déterrer, sorte de trésor enfoui que les travaux sur les textes permettraient de mettre au jour. La question des valeurs est, nous semble-t-il, inséparable des démarches mises en œuvre dans toutes les activités d'apprentissage (Fourtanier \& Langlade, 2000, p. 14).

18 Les propositions de démarches et d'activités qui suivent s'inspirent et rendent compte en partie de la séquence sur La Belle et la Bête présentée supra.

\section{Susciter des échanges intergénérationnels}

19 Afin de faire éprouver le patrimoine littéraire comme un héritage commun, il me semble intéressant de susciter un dialogue entre les élèves et leurs familles par l'intermédiaire d'un questionnaire portant sur une œuvre patrimoniale et sur les traces déposées par le récit (et ses OSS) dans la mémoire des lecteurs (et des spectateurs). Dans la classe témoin, un tel questionnaire a été proposé par l'enseignante à ses élèves au sujet du conte de madame Leprince de Beaumont, avant d'en entamer l'étude. Les élèves ont donc répondu en classe, individuellement et par écrit, aux questions suivantes : "Connais-tu La Belle et la Bête ? », « Peux-tu résumer l'histoire? ", " Sais-tu de quand date cette histoire?", "Selon toi, quelle est la morale de cette histoire?", "Comment as-tu connu cette histoire (quand, où, comment, grâce à qui...) ?» Le jour même, chaque élève a interviewé ses proches (parents, et, pour certains, grandsparents ou frères et sœurs) sur la base de ce même questionnaire (réponses écrites). Le jour suivant, les réponses apportées au questionnaire par les élèves et par leurs familles ont été dépouillées, mises en commun et comparées en classe, sous la forme d'un tableau qui visait à mettre en évidence plusieurs caractéristiques de l'œuvre patrimoniale.

20 1. L'œuvre patrimoniale est un bien commun, dont la connaissance, largement partagée, se joue des différences générationnelles : à la question « Connais-tu La Belle et la Bête?", on obtient seulement deux réponses négatives sur les vingt-trois questionnaires récupérés, et, du côté des élèves également, deux sur vingt-et-un seulement disent ne pas connaitre cette histoire. Les souvenirs de l'histoire sont souvent très lacunaires, vagues et divergents, et témoignent d'une réception et d'une appropriation personnelles de l'œuvre, largement reconfigurée par le travail de la mémoire ; ils se prêtent cependant à l'échange, et les souvenirs de l'œuvre instaurent un lieu commun où l'on peut se retrouver en dépit des différences intersubjectives.

21 2. L'œuvre patrimoniale est un héritage du passé : la rédaction du conte est située par les parents qui ont répondu à la question «Sais-tu de quand date cette histoire? ? au XVIe siècle (une réponse), au XVIIe (une réponse), au XVIIIe (neuf réponses), au XIXe (deux réponses), au XXe siècle (une réponse) ${ }^{5}$. Informés par l'enseignante de l'année de publication du texte (1756, dans le recueil intitulé le Magasin des enfants ${ }^{6}$ ), les élèves constatent la faculté qu'ont les œuvres du patrimoine à circuler dans le temps et à 
tisser de ce fait des liens intergénérationnels ; ils perçoivent qu'un texte patrimonial est bien « du passé dans le présent » (Louichon, 2015a).

3. Les courroies de transmission de l'œuvre patrimoniale ne se cantonnent pas à la sphère scolaire et impliquent l'entourage proche, sur le mode de la dévolution matrilinéaire et patrilinéaire. À la question «Comment as-tu connu cette histoire?", huit parents évoquent le rôle de leurs propres parents dans cette transmission, quel qu'en soit le support matériel (textuel ou audiovisuel) : «enfant avec mes parents sur cassette vidéo louée au vidéoclub", "mes parents me la lisaient avant de dormir ", "j'ai connu cette histoire quand j'étais petite fille dans un livre de contes de fées offert par mes parents ", « le film avec Jean Marais en étant adolescente avec mes parents", " je l'ai vue à la télé grâce à mes parents ", " c'est ma maman qui me l'a fait connaitre ", "je l'ai connue enfant, chez moi, grâce à ma maman ", "c'est maman qui m'avait acheté la cassette ». Du côté des élèves, Alexy doit sa connaissance du conte à " [sa] mère, [sa] tata et [sa] mamie ", tandis que Matthias dit connaitre l'histoire «car [sa] sœur a regardé plusieurs fois» le film. Il est intéressant de constater que cette circulation ne s'opère pas à sens unique : une réponse signale que ce sont les enfants qui transmettent l'œuvre à leurs parents (un parent dit avoir découvert La Belle et la Bête « à la télé [...], par [ses] enfants, avec le film »).

Autour des textes du patrimoine et des objets sémiotiques secondaires qu'ils génèrent se rassemblent ainsi des lecteurs, spectateurs et auditeurs de tous âges. En suscitant des échanges entre enfants et parents, l'exercice du questionnaire révèle que la lecture de ces textes peut faire sens pour le sujet, ici et maintenant, et revêtir des enjeux à la fois personnels et collectifs, qui s'étendent bien au-delà de la seule injonction scolaire.

Les élèves ont été particulièrement sensibles aux échanges suscités par le conte et l'interview, comme le confirme le "méta-questionnaire " écrit auquel ils ont dû répondre par la suite. Il leur a été tout d'abord demandé comment ils avaient mené l'interview de leurs proches. Sur les vingt-deux élèves qui ont répondu à ce second questionnaire, quatorze signalent qu'ils ont eux-mêmes posé oralement les questions à leurs parents; quatre élèves seulement ont indiqué que leurs parents ont pris connaissance et répondu au questionnaire en leur absence. Pour deux d'entre eux, c'est d'ailleurs la raison pour laquelle ils n'ont pas apprécié l'exercice de l'interview : « car je n'étais pas à côté de mes parents quand ils ont fait le questionnaire ", "car je n'étais pas là quand ils ont répondu au questionnaire ", écrivent-ils l'un et l'autre. Sur les dixhuit élèves qui disent avoir aimé soumettre le questionnaire initial à leurs familles, quatre ont été tout particulièrement sensibles à la dimension investigatoire de l'exercice - "ça faisait un peu enquête policière », précise Loris ; trois font valoir leur intérêt personnel pour l'histoire de La Belle et la Bête; neuf mettent en avant la relation privilégiée qui a pu s'établir avec leurs parents à l'occasion de l'interview : «c'était un très bon moment, en famille en plus ", écrit Baptiste ; « c'est rigolo de faire rappeler à ses parents l'histoire de La Belle et la Bête ", ajoute un autre enfant; un troisième précise que l'interview était un bon prétexte pour recueillir les souvenirs de sa mère et «la tenir devant un café »; on peut conclure avec Sofiène, qui apprécie d'avoir « passé un moment avec [ses] parents ».

Le questionnaire me parait propre à faire prendre conscience que le patrimoine littéraire est un legs reçu en partage, un élément cohésif ; sa connaissance, qu'elle soit due à l'œuvre originale ou à ses OSS, unit les élèves et leurs parents, comme elle les unit aux lecteurs des siècles passés. Le dispositif introduit en outre une forme de fraternité 
en éducation en engageant les parents dans l'école via l'échange avec l'enfant; il permet également d'accueillir en classe des objets généralement assignés à résidence hors de la sphère scolaire.

\section{Accueillir les objets et les pratiques ordinaires de lecture}

Il me semble en effet que l'atout des œuvres patrimoniales dans une tentative d'éducation à la fraternité est qu'elles invitent à introduire en classe des objets et des pratiques culturelles juvéniles qui généralement n'y ont pas accès. Les textes du patrimoine pourraient ainsi constituent des objets transitionnels entre la sphère scolaire et la sphère familiale, alors que «les deux espaces se constituent d'abord symboliquement de manière antinomique » (Grossmann, 2006, p. 21).

Caractérisée par le double mouvement de réception-transmission déjà évoqué, l'œuvre patrimoniale se signale en outre par sa faculté à générer des OSS qui témoignent que la réception de l'œuvre patrimoniale continue de s'opérer au présent, en même temps qu'ils constituent un indice fort de sa patrimonialité - il suffit d'entrer la requête $L a$ Belle et la Bête dans un moteur de recherche, en particulier dans Google Images, pour constater la multiplicité des OSS suscités par ce conte. Il me parait intéressant pour plusieurs raisons d'intégrer ces objets dans l'étude des textes patrimoniaux en classe, dans le cadre par exemple de parcours de lecture multimodaux. D'une part, ces «produits dérivés » - et j'emploie l'expression sans intention péjorative - sont souvent le premier et le seul contact des enfants et de leur famille avec l'œuvre patrimoniale dont ils émanent. Ainsi le questionnaire révèle que quatorze des vingt-et-un parents qui connaissent $\mathrm{La}$ Belle et la Bête l'ont découvert dans une version cinématographique (cinq mentionnent explicitement le dessin animé des studios Disney), quatre seulement grâce au livre; trois ne se prononcent pas.

$\mathrm{Du}$ côté des élèves, tous mentionnent dans leurs réponses au questionnaire les adaptions cinématographiques parmi les "courroies de transmission» assurant la connaissance de l'histoire. En ne tenant compte que du premier support évoqué, sur les dix-neuf enfants qui disent connaitre La Belle et la Bête, quatorze (dont cinq mentionnent explicitement le dessin animé) le doivent à l'une ou l'autre de ces adaptations. Cinq affirment qu'ils ont découvert cette histoire dans un livre (pour un élève, il semble que ce livre soit un album présentant la version des studios Disney et non le texte original). Deux enfants mentionnent aussi la comédie musicale adaptée du long métrage des studios Disney et un autre une transposition théâtrale que je ne connais pas.

Il serait donc dommage de ne pas recevoir ces OSS en classe alors même qu'une large majorité d'élèves et de parents leur doivent leur connaissance liminaire de l'œuvre patrimoniale. Il ne s'agit évidemment pas de tomber dans une forme de démagogie pédagogique, ni de donner à entendre que le dessin animé des studios Disney vaut le film de Jean Cocteau ou l'œuvre littéraire mais d'inclure ces objets dans un dispositif didactique tenant compte des spécificités de l'œuvre patrimoniale et en particulier de sa faculté à engendrer des discours seconds. Il revient précisément à l'école d'outiller les élèves afin qu'ils soient capables de distinguer l'œuvre originale de ses hypertextes et d'opérer un tri entre les adaptations « ordinaires », les adaptations " réductrices » et les adaptations " créatrices ", de les situer sur l'" échelle de légitimité de la littérature » (Pintado, 2008, p. 27). C'est d'autant plus nécessaire que les adaptations les plus 
indigentes sont généralement adressées à des enfants qui ne fréquentent pas les lieux traditionnels de lecture, bibliothèques et librairies, ce qui contribue à accroitre les inégalités socioculturelles. «Puisque l'industrie éditoriale détourne les textes au profit d'objectifs financiers, l'école doit détourner ces objets afin de leur faire servir des objectifs culturels et littéraires ", note avec justesse Brigitte Louichon (2008, p. 24).

Une école fraternelle a donc tout intérêt à accueillir les objets relevant de la culture dite populaire ou de masse - bandes dessinées, dessins animés, jeux vidéo, etc., "qui constituent désormais l'environnement culturel quotidien des jeunes d'aujourd'hui » (Baudelot, Cartier \& Detrez, 1999, p. 62) -, ainsi que les pratiques qu'ils suscitent, en veillant à « se garder de deux dérives, celle consistant à analyser une œuvre pour mieux la dévaloriser, l'inférioriser, et finalement l'exclure de la classe, et celle qui, au contraire, valorise le "produit" à l'excès » (Prévost, 2011, p. 105).

Il ne s'agit donc pas, insistons-y, de donner à entendre que tout se vaut, mais de réduire le « hiatus entre culture familiale et culture scolaire » (Demougin, 2012, p. 231) souvent signalé, pour problématiser la lecture et l'étude des textes intégrés avec leurs OSS dans des parcours de lecture multimodaux (Gennaï, 2018a), pour faire des pratiques culturelles personnelles des élèves et des biens qu'elles mobilisent un objet de réflexion et de discours critiques.

\section{Fonder une « fraternité interprétative »}

Pour Michèle Petit, " porter dans l'espace de la classe certaines des réflexions surgies à l'occasion d'une lecture et apprendre à en débattre, à les formaliser, peut probablement contribuer à l'élaboration d'une citoyenneté [...] (Petit, 2002, p. 131). La confrontation des textes patrimoniaux et des OSS qu'ils génèrent peut alimenter des séances de débat interprétatif, qui font de la classe une communauté interprétative, voire fondent une " fraternité interprétative ", pour reprendre l'expression de Françoise Demougin (2012, p. 238), où sont négociés les sens possibles du texte dans l'échange et la socialisation des subjectivités. Débattre des textes « en développant son expression, la formulation de ses opinions, dans des échanges oraux » contribue ainsi selon les programmes pour le cycle de consolidation à la "formation de la personne et du citoyen " (l'un des domaines du nouveau socle commun de connaissances, de compétences et de culture). Pour instaurer pleinement cette fraternité lettrée, il me semble qu'il convient de ne pas envisager la lecture sous le seul angle des savoirs formels qu'elle met en jeu et de " permettre la réception sensible des œuvres littéraires " (Bulletin officiel spécial n 11 du 26 novembre 2015), d'accueillir l'expression des lectures singulières, leur dimension psychoaffective, les émotions lectorales, les manifestations de "la relation émotionnelle que le lecteur entretient avec le monde fictionnel et les acteurs du récit que sont les personnages » (Larrivé, 2015).

Certes, le dispositif du débat interprétatif n'est pas réservé aux seules œuvres patrimoniales. Cependant, dans la mesure où elles ont suscité (et l'on a signalé que c'était là un des indices de leur patrimonialité) de nombreux métatextes, au sens où Genette (1982, p. 10) emploie ce terme, elles se prêtent davantage encore que les textes contemporains au débat: les commentaires, préfaces, critiques, notices, etc., rédigés depuis l'époque de publication et jusqu'à nos jours, témoignent tous d'une réception à la fois singulière et historiquement située des œuvres. Dès lors, il peut être intéressant d'inviter les élèves à confronter leurs lectures d'un texte du patrimoine à celles qu'en 
ont faites jadis d'autres lecteurs ; on élargit ainsi dans le temps et dans l'espace le cercle de la "fraternité interprétative », tout en recontextualisant l'œuvre étudiée.

Par ailleurs, je pense qu'on peut montrer aux élèves que la fraternité lectorale qui gravite dans l'orbite des œuvres patrimoniales s'étend bien au-delà des frontières nationales. Un parcours de lecture prenant pour point de départ le texte de JeanneMarie Leprince de Beaumont révèle que le conte-type a donné naissance à d'innombrables variantes qui traversent l'espace et le temps - l'anthologie de Fabienne Morel et Gilles Bizouerne illustrée par Delphine Jacquot et intitulée Les histoires de la Belle et la Bête racontées dans le monde (Paris, Syros, 2008) présente des versions russe, égyptienne, bretonne, écossaise, japonaise, norvégienne et canadienne du conte. La notion de patrimoine, d'abord restreinte au strict cadre national et associée à l'expression du "génie » français (Houdart-Merot, 2012, p. 32), révèle sa dimension mondiale. Les mythes, les épopées homériques, les contes d'Andersen et ceux des Mille et Une Nuits, etc., font partie du patrimoine universel de l'humanité : si ces œuvres confrontent les lecteurs contemporains à l'altérité, à des axiologies et des systèmes de valeurs autres, elles n'en expriment pas moins, en dépit de leur éloignement dans le temps et dans l'espace et de contextes de production très différents, les grandes interrogations, les aspirations, les craintes qui travaillent chacun des membres de «la grande famille humaine» (Bidar, 2015, p.68), passée, présente et à venir. Une éducation à la "fraternité universelle " (ibid.) peut prendre appui sur ce que ces œuvres révèlent de notre humaine condition.

\section{Susciter l'empathie}

Dans le cadre de l'expérimentation menée dans la classe de CM2, des travaux d'écriture tentent de tirer profit des ressources du patrimoine littéraire au service d'une éducation à la fraternité. La rédaction suivie du journal intime de la Belle engage les élèves dans une écriture en je fictif. Ce travail d'écriture s'appuie sur une lecture projective et enrôle les élèves dans un processus d'empathie fictionnelle. Zanna (2010) démontre que les enfants et adolescents en grande détresse psychologique, sociale et scolaire peinent précisément à entrer en empathie avec autrui et que les apprentissages intellectuels semblent corrélés aux compétences psychosociales. Tout en donnant matière à une interprétation plus réfléchie des textes, ces activités de lecture et d'écriture qui mobilisent l'empathie fictionnelle peuvent favoriser le décentrement de soi, l'accès à d'autres formes d'empathie, psychologique et cognitive, et développer les phénomènes de communication intersubjective permettant de comprendre ce que ressent autrui :

tout exercice sollicitant la communication empathique avec les personnages de la fiction permet de développer non seulement l'aptitude à l'empathie fictionnelle des lecteurs-scripteurs, mais aussi l'aptitude sociale de ces élèves à la communication empathique avec leurs pairs. Or il s'agit bien d'une des finalités psychoaffectives de l'enseignement de la littérature (Larrivé, 2015).

Enfin, dans le cadre d'un autre projet d'écriture au long cours, les élèves ont rédigé leur propre adaptation de La Belle et la Bête, sous la forme d'une pièce de théâtre, jouée à l'occasion de la fête de fin d'année devant les familles. Ils ont également réalisé une adaptation du conte de Marie Leprince de Beaumont : un petit album lu aux enfants des classes maternelles de l'école. Ainsi, après avoir échangé avec leurs ainés autour de l'œuvre patrimoniale, les élèves poursuivront l'échange intergénérationnel avec leurs 
puinés. Chacun deviendra à son tour acteur du geste patrimonial de conservation et de transmission - acteur et auteur, c'est-à-dire auctor, "celui qui augmente " (du latin augere), ce qui correspond bien à la définition des classiques selon Roland Barthes (2002, p. 59): «des trames, des ébauches, des ébauches où l'on peut indéfiniment ajouter $»$.

\section{Conclusion}

Comment faire du patrimonium un fratrimonium? C'est en s'éprouvant, non en s'énonçant, que la fraternité se révèle désirable et se transmet - "On n'apprend plus l'art de vivre ensemble par la seule autorité des leçons de morale et de la discipline » (Dubet, 2014, p. 94). Les hypothèses et les propositions de mises en œuvre didactique évoquées ici, et dont il reste à éprouver la pertinence par des recherches empiriques, visent ainsi à faire percevoir et à faire expérimenter aux élèves ce qui se joue dans le processus de patrimonialisation: conservation et transmission d'un bien commun, réception partagée au présent d'une œuvre du passé. Grâce aux interactions suscitées entre les élèves et leurs parents par les interviews, les œuvres du patrimoine littéraire mondial peuvent se révèler comme une monnaie d'échange intergénérationnelle, qui a également cours et circule entre pairs, et ignore les frontières temporelles, nationales, linguistiques et culturelles.

L'enseignement de la littérature patrimoniale a sans doute son rôle à jouer dans l'éducation à la fraternité à condition que s'opère l'inclusion scolaire, autour de ces œuvres, de situations et de pratiques discursives (en l'occurrence l'interview, où l'élève prend le rôle, pour lui inhabituel, de l'interrogateur), d'objets (les adaptations de l'œuvre qui relèvent de la culture populaire) et d'interlocuteurs (parents, aïeuls, fratrie) que l'école peine généralement à accueillir. Le patrimoine littéraire peut œuvrer à une éducation à la fraternité dans la mesure où les élèves éprouveront à l'école et hors de l'école le partage qu'il fonde et qui le fonde en tant qu'objet en libre circulation - partage des œuvres littéraires dans les OSS qu'elles génèrent, partage intergénérationnel auprès des ainés et des puinés, partage entre pairs également au sein de la «fraternité interprétative » de la classe.

Si la littérature patrimoniale en particulier et la littérature en général peuvent contribuer à une éducation à la fraternité, il se pourrait également que l'instauration d'une communauté lectorale contribue à développer le gout pour la lecture. Dans une tentative d'identification et d'explication des "ratés" du système scolaire en la matière, Catherine Frier et Marie-Cécile Guernier attribuent le manque d'appétence des élèves du secondaire pour les livres prescrits dans le cadre du cours de français à "l'incapacité de l'école à prendre en compte l'individu, en travaillant par exemple davantage le lien lecture scolaire/lecture extrascolaire » - et, ajouterai-je, le lien entre les lectures et les pratiques scolaires d'une part et l'ensemble des autres objets et pratiques culturelles extrascolaires :

nous savons bien à quel point la reconnaissance des pratiques individuelles des élèves peut constituer l'amorce d'un « habitus ", en permettant notamment au sujet de se placer dans une "communauté de lecteurs", incarnée "dans des gestes, des espaces, des habitudes ». C'est à ce prix que « la lecture pourrait alors (re)devenir le lieu d'un échange, du partage social d'un message » (Frier \& Guernier, 2007, p. 137, citant Privat, 1993). 


\section{BIBLIOGRAPHY}

Ahr, S. \& Denizot, N. (Dir.). (2013). Les patrimoines littéraires à l'école. Usages et enjeux. Namur : Presses universitaires de Namur.

Bard, C. (2010). Une histoire politique du pantalon. Paris : Éditions du Seuil.

Barthes, R. (2002). Plaisir aux Classiques. In Cuuvres complètes, tome I. Paris : Seuil.

Baudelot, C., Cartier, M. \& Detrez, C. (1999). Et pourtant ils lisent... Paris : Éditions du Seuil.

Bidar, A. (2015). Plaidoyer pour la fraternité. Paris : Albin Michel.

Bishop, M.-F. (2015). Lire, comprendre, interpréter ? Quels objectifs pour la lecture des textes littéraires à l'école élémentaire, en France, depuis la fin du XIXe siècle ? In A. Petitjean (Ed.), Didactiques du français et de la littérature (pp. 365-386). Metz : Université de Lorraine, Crem, « Recherches textuelles », $\mathrm{n}^{\circ} 14$.

Bishop, M.-F. \& Belhadjin, A. (Dir.). (2015). Les patrimoines littéraires à l'école. Tensions et débats actuels. Paris : Champion.

Calvino, I. (1991). Pourquoi lire les classiques. Paris : Éditions du Seuil.

Condorcet, N. de (1795). Esquisse d'un tableau historique des progrès de l'esprit humain.

Demougin, F. (2012). Lire un texte patrimonial à l'école primaire : de lectures en lecteurs. L'exemple du « Dormeur du val » en CM2 (Réseau Ambition Réussite). In I. de Peretti et B. Ferrier (Eds.), Enseigner les « classiques » aujourd'hui. Approches critiques et didactiques (pp. 231-245). Bruxelles : Peter Lang.

Denizot, N. (2015). Patrimonialisation de la littérature (XIXe siècle-XXe siècle). In M.-F. Bishop et A. Belhadjin (Eds.), Les patrimoines littéraires à l'école. Tensions et débats actuels (pp. 109-121). Paris : Champion.

De Peretti, I. \& Ferrier, B. (Dir.). (2012). Enseigner les « classiques » aujourd'hui. Approches critiques et didactiques. Bruxelles : Peter Lang.

Derrida, J. (1986). Schibboleth, pour Paul Celan. Paris : Galilée.

De Tanoüarn, G. (2018). Le prix de la fraternité. Paris : Tallandier, coll. « Essais ».

Donnat, O. (2009). Les pratiques culturelles des français à l'ère numérique. Paris : La Découverte Ministère de la culture et de la communication.

Dubet, F. (2014). La préférence pour l'inégalité. Comprendre la crise des solidarités. Paris : Éditions du Seuil.

Faucher, C. (2015). Entrevue avec Philippe Meirieu. In Réflexions. Fondement de l'enseignement des arts au Québec, Vision, numéro hors série. Québec : aqésap.

Fourtanier, M.-J. \& Langlade, G. (2000). Enseigner la littérature. Actes du colloque Enjeux didactiques des théories du texte dans l'enseignement du français. Paris, Toulouse : Delagrave, CRDP MidiPyrénées.

Frier, C. \& Guernier, M. C. (2007). Paroles de lecteurs : et si les usages scolaires empêchaient de pratiquer la lecture ? Repères, 35, 117-138. 
Gennaï, A. (2018a). Parcours multimodal de lecture dans une oeuvre du patrimoine au cycle 3. Dans J.-C. Chabanne (dir.), Enseigner la littérature en dialogue avec les arts. Confrontations, échanges et articulations entre approches didactiques (pp. 139-157). Namur : Presses universitaires de Namur.

Gennaï, A. (2018b). Patrimoine littéraire et formation éthique dans les textes officiels pour l'école élémentaire (cycle 3) en France. Repères, 58, 63-79.

Grossmann, F. (2006). Logiques sociales et clivages culturels dans les lectures partagées. In C. Frier (Dir.), Passeurs de lecture. Lire ensemble à la maison et à l'école (pp. 19-43). Paris : Retz.

Heinich, N. (2009). La fabrique du patrimoine. De la cathédrale à la petite cuillère. Paris : Éditions de la Maison des sciences de l'homme.

Houdart-Mérot, V. (2012). Qu'est-ce qu'un classique? Qu'est-ce qu'une œuvre patrimoniale ? In I. de Peretti et B. Ferrier (Eds.), Enseigner les « classiques » aujourd'hui. Approches critiques et didactiques (pp. 23-36). Bruxelles : Peter Lang.

Jacquet-Francillon, F. (2015). Le passé n'est plus ce qu'il était. Sens et fonctions des pratiques contemporaines du patrimoine. In M.-F. Bishop et A. Belhadjin (Eds.), Les patrimoines littéraires à l'école. Tensions et débats actuels (pp. 59-71). Paris : Champion.

Larrivé, V. (2015). Empathie fictionnelle et écriture en « je » fictif. Repères, 51, 157-176. http:// reperes.revues.org/913.

Louichon, B. (2015a). Le patrimoine littéraire : du passé dans le présent. In M.-F. Bishop et A. Belhadjin (Eds.), Les patrimoines littéraires à l'école. Tensions et débats actuels (pp. 93-106). Paris : Champion.

Louichon, B. (2015b). Le patrimoine littéraire : un enjeu de formation. Tréma : vol. 43 Culture humaniste et formation des enseignants (pp. 23-31).

Louichon, B. (2008). L'adaptation : grandeur et misère du patrimoine littéraire. In Adapter des œuvres littéraires pour les enfants (pp. 11-26). Grenoble : CRDP de l'Académie de Grenoble.

Petit, M. (2002). Éloge de la lecture. La construction de soi. Paris : Belin.

Pintado, C. (2008). Traduction, détournement et/ou recréation. L'adaptation des Contes de Perrault. In Adapter des œuvres littéraires pour les enfants (pp. 27-44). Grenoble : CRDP de l'Académie de Grenoble.

Prévost, C. (2011). Quelle place pour des « produits culturels de masse » dans la classe de français? Le français aujourd'hui, 172 (1), 103-112.

Privat, J.-M. (1993). L'institution des lecteurs. Pratiques, 80, 7-34.

Rey, A. (Dir.). (1992). Dictionnaire historique de la langue française. Paris : Le Robert.

Zanna, O. (2010). Restaurer l'empathie chez les mineurs délinquants. Paris : Dunod.

\section{NOTES}

1. École André-Galan, classe de CM2 d'Élisabeth Gorses, PEMF.

2. C'est moi qui souligne.

3. Pour une étymologie erronée mais qui n'en éclaire pas moins la fonction symbolique du patrimoine, voir les propos de Philippe Meirieu : «J'aime bien rappeler l'étymologie de ce terme selon Francis Imbert, qui veut dire sym-boilos, "sans le javelot", c'est-à-dire sans la violence, sans le passage à l'acte. La capacité de symboliser, c'est la capacité de vivre dans un univers où on ne 
passe pas à l'acte, d'accéder à cette espèce de geste premier de l'humain que l'on trouve si bien exprimé, par exemple, dans la métaphore des chevaliers de la Table ronde (déposer son épée à l'entrée)» (cité par Faucher, 2015, p. 24).

4. Si «l'hypertextualité est une relation d'imitation et de transformation entre textes", la transfictionnalité, désigne "une relation de migration (avec la modification qui en résulte presque immanquablement) de données diégétiques » (Saint-Gelais, 2011, p. 10-11). Il s'agit « des récits et des histoires qui font migrer un élément fictionnel (souvent le personnage), qui racontent autre chose à son propos. [...] Ainsi, lorsque Tim Burton met en scène une Alice de 19 ans ou que Régis Loisel invente la première enfance de Peter Pan, ils ne réécrivent ni d'adaptent les œuvres originales, ils font migrer les personnages et un certain nombre de données fictionnelles dans une autre histoire » (Louichon, 2015b, p. 26).

5. Les autres ne répondent pas à la question.

6. On sait que la première version écrite de l'histoire est l'œuvre de Gabrielle-Suzanne de Villeneuve ; elle figure dans un recueil intitulé La Jeune Américaine et les contes marins et publié en 1740.

\section{ABSTRACTS}

How can the literary heritage contribute to the promotion of fraternity at school? This paper examines how, rather than the values that those texts thematize, their very nature, the process by which they achieve the status of literary heritage and the modes of teaching can provide a basis for educating to fraternity. We will also explore how it is possible for the pupils to experience the processes of transmission and intergenerational exchange of a shared legacy, processes which are constituent elements of this heritage.

En quoi les textes du patrimoine peuvent-ils contribuer à l'éducation à la fraternité à l'école ? Davantage que les valeurs thématisées par ces textes, ce sont la nature même de l'objet patrimonial, le processus de patrimonialisation et les modalités d'enseignement qui peuvent fonder une éducation à la fraternité. Il s'agit alors de faire éprouver aux élèves la dimension patrimoniale des œuvres, fondée sur la constitution d'un héritage commun dans un "double mouvement de réception-transmission » (Denizot, 2015, p. 110).

\section{INDEX}

Mots-clés: école primaire, fraternité, patrimoine littéraire

Keywords: fraternity, literary heritage, primary school

\section{AUTHOR}

\section{ALDO GENNAÏ}

Université de Montpellier - LIRDEF, ALFA (EA 3749) 